\title{
Variations
}

Variations

Revue internationale de théorie critique

$21 \mid 2018$

L'industrie de la culture : version originale

\section{Eviter l'amour du sable qui crisse: propositions pour une économie politique critique des industries de la culture et de la communication}

Jacob Matthews

\section{OpenEdition}

Journals

Édition électronique

URL : http://journals.openedition.org/variations/922

DOI : $10.4000 /$ variations. 922

ISSN : 1968-3960

Éditeur

Les amis de Variations

Référence électronique

Jacob Matthews, «Eviter l'amour du sable qui crisse: propositions pour une économie politique critique des industries de la culture et de la communication 》, Variations [En ligne], 21 | 2018, mis en ligne le 05 avril 2018, consulté le 05 mai 2019. URL : http://journals.openedition.org/variations/922 ; DOI : 10.4000 /variations.922

Ce document a été généré automatiquement le 5 mai 2019.

Les ami•e•s de Variations 


\title{
Eviter l'amour du sable qui crisse: propositions pour une économie politique critique des industries de la culture et de la communication
}

\author{
Jacob Matthews
}

«Francfort, 18 septembre 1962. Je tenais entre les mains un exemplaire imprimé des Passages de Benjamin, ou bien parce qu'il les avait tout de même achevés, ou bien parce que je les avais reconstitués à partir des brouillons. Je les lisais avec amour. Un titre annonçait "deuxième partie" ou "deuxième chapitre". En dessous, cet exergue :

"Quel wagon de tramway aurait l'insolence d'affirmer qu'il roule exclusivement pour l'amour du sable qui crisse ? Robert August Lange, 1839." " Theodor W. Adorno, Mes rêves

\section{Introduction}

1 Ce texte s'appuie sur les conclusions de recherches menées au cours des dix dernières années sur les évolutions des industries de la culture et de la communication dans le contexte de l'expansion des technologies d'information et de communication numériques (TICN). Ces travaux ont d'abord montré comment les acteurs du web concourent à une mise en système renforcée des ces industries, tout en favorisant leur rapprochement avec les industries des biens et services de consommation courante. En second lieu, ils ont questionné la portée idéologique du développement du web 2.0 et de la «culture participative $»^{1}$. Des enquêtes portant sur les plateformes web de production et de financement de projets culturels ont permis à la fois de développer ces deux points et d'approfondir l'analyse des représentations idéologiques de la «créativité » et de la 
«collaboration» qui accompagnent les usages de ces dispositifs, qu'elles émanent de sources industrielles, institutionnelles, médiatiques, ou encore des discours d'usagers ${ }^{2}$. Il ne s'agit pas de reproduire ici la synthèse critique de ces travaux réalisée au titre de mon mémoire d'habilitation à diriger des recherches, mais de développer une réflexion figurant en marge de ce texte. Celle-ci s'appuie sur un postulat double.

2 Premièrement, les productions ${ }^{3}$ des industries de la culture et de la communication, tout comme les discours accompagnant les usages de TICN qui leur sont désormais massivement associées, constituent des «formes culturelles». Étant donnée la place centrale que cette notion occupe dans mon exposé, il convient d'emblée de préciser le sens qui lui est conféré par certains travaux d'économie politique critique de la communication: celui-ci renvoie à la proposition selon laquelle les productions intellectuelles, symboliques, constituent - en dernière instance - des formes culturelles des relations de production matérielles qui soutiennent les sociétés dans lesquelles émergent ces productions. Le degré d'autonomie de formes culturelles, vis-à-vis des relations de production matérielles, a constitué un objet récurent de recherches matérialistes. Ceci est caractéristique des travaux de Raymond Williams ${ }^{4}$ interrogeant la possibilité de formes culturelles oppositionnelles, face à ce qu'il nomme la culture dominante effective. Inversement, se pose la question du degré d'effectivité matérielle de formes culturelles - autrement dit, comment des représentations idéologiques seraient à même de consolider ou, inversement, d'ébranler les relations de production elles-mêmes.

Deuxièmement, il parait utile que l'économie politique critique de la communication se (re)saisisse de cette question, à l'heure où se répandent les discours de la « créativité » et de la "collaboration", et alors que les usages des TICN requièrent une participation de plus en plus active et bruyante aux processus de valorisation capitaliste - que certains nomment «travail numérique » (digital labour). D’un point de vue épistémologique, le problème s'épaissit quelque peu, dès lors qu'on admet que les recherches critiques doivent à la fois déconstruire ces représentations idéologiques, et questionner plus largement les produits des sciences sociales en tant que formes culturelles eux-mêmes, ayant des relations essentiellement médiates avec les réalités matérielles qu'ils représentent $^{5}$. Il ne saurait dès lors être question de se soustraire à l'analyse des médiations qui les lient à la base économique de la société, sauf à rompre avec l'ambition dialectique de la théorie critique.

Ce texte a pour but de réexaminer une série de propositions théoriques fondamentales sur lesquelles s'appuient ces deux hypothèses, en illustrant comment elles peuvent être utiles (et complémentaires) pour appréhender à la fois les évolutions présentes des industries de la culture et de la communication et, plus encore, pour contribuer au positionnement réflexif qu'exige la recherche critique dans ce champ.

\section{Formes de pensée et rapports de production}

Dans un texte rédigé à la fin des années 1970, Contribution to a political economy of masscommunication, Nicholas Garnham développe un point qui peut a priori sembler anecdotique, mais dont on ne devrait pas sous-estimer l'importance pour les recherches qui s'efforcent aujourd'hui de réintroduire ou de réactualiser la pensée critique dans le champ des TICN - notamment via le débat autour du digital labour ${ }^{6}$. Le point en question part d'un souhait de clarifier la confusion que laisse planer Raymond Williams, dans son texte précité de 1973, entre le «matériel " et l' «économique»; je me permets de le 
reproduire quasiment in extenso étant donné qu'une synthèse risquerait de rendre la démonstration moins intelligible :

Insofar as historical materialism is materialist, it is based upon the postulates that Williams outlines. But insofar as it is historical, it is concerned to analyse the specific and shifting modes of this fundamental material relation, all of which are forms of that relation. In particular, it is postulated that any form of extended social relationship depends upon the extraction and distribution of material surplus and the means by which this is achieved is thus the central determining characteristic of any social formation. [...] Thus we must distinguish two types of form, a social form which is a series of material relations that, insofar as they operate unconsciously, can be abstractly analysed and determined with the precision of natural science, and a cultural form which, while it entails a material support, is not itself material and which has an essentially mediated relationship with the material reality it represents. Indeed, there is an essential divide between these distinct formal realms, the existence of which allows ideology to enter, because it allows denial and the lie, both of which depend upon a relationship which is not determinant. However, this autonomy is bought at the cost of a loss of real or material effectivity. Cultural forms only become effective when they are translated into social forms which do have material effectivity. [...] To return to the level of social forms, the economic is a specific historical form of the social relations of production and distribution. It is the form these relations take in a social formation within which commodity exchange is dominant. [...] What Marx argues in Capital is that the real historical transition to capitalism involves a move from a system of social relations and domination based upon the direct physical control of landed property and people to one based upon the increasingly indirect control through commodity exchange and, in particular, through the exchange of the commodity of labour power, and that this real historical process is a real process of social abstraction which thus requires appropriate theoretical abstraction for its analysis. [...] It is the real existence of this abstract economic level of extended commodity production that allows for the development of an increasing division of labour and thus for the development of the specific superstructural forms of capitalism. Thus the relative autonomy of the superstructure is a real and increasingly central characteristic of capitalism, but it is itself determined at the level of the economic and ultimately it is a form, at two levels of mediation, of a material relation which also remains determinant in and through the economic. ${ }^{7}$

6 Dans cet article Garnham affirme la validité générale du modèle base / superstructure ${ }^{8}$, tout en proposant, à la suite de cet extrait, de contribuer à l'analyse du développement historique des industries de la culture en tant que processus spécifique d'industrialisation de la superstructure, sans pourtant jamais se départir de l'intuition centrale de Marx concernant le mode de production capitaliste, à savoir «sa dynamique de généralisation et d'abstraction; la pression de tout réduire à l'équivalence de la valeur d'échange. ॥" Le caractère absolument fondamental de cette abstraction "incarnée » que constitue la forme marchandise et sa valeur d'échange n'avait évidemment pas échappé aux auteurs de La dialectique de la raison, mais ce sont les travaux moins connus du philosophe Alfred Sohn-Rethel qui explorent le plus finement les soubassements et conséquences de cette intuition centrale.

7 D'après Alfred Sohn-Rethel ${ }^{10}$, le processus matériel et historique par le biais duquel émerge puis se consolide la forme marchandise peut être analysé à partir de l'acte matériel d'échange entre deux biens et, a fortiori, de l'acte d'échange d'un bien pourvu d'une valeur d'usage contre un objet particulier dépourvu de valeur d'usage, la monnaie frappée (qui émerge au VIIème siècle avant l'ère chrétienne). ${ }^{11}$ Il souligne que le trait dominant de la forme marchandise est son caractère abstrait: la marchandise émerge 
dans l'échange et n'existe que dans la pensée des hommes. Dans l'acte de l'échange marchand, la matérialité de la valeur d'usage d'un bien est comme suspendue, au profit de la valeur d'échange inaltérable et intemporelle de la marchandise. Ce que ce théoricien nomme « abstraction-marchandise » repose donc sur une action qui est matériellement inscrite en un lieu et un moment donnés, et qui a pour conséquence à la fois l'abstraction de la valeur d'usage d'un bien (l'échange supprime localement et temporairement celleci), et l'émergence d'une forme abstraite, qui n'existe pas dans la nature ou dans la réalité matérielle, qui n'a donc d'existence que dans la pensée de l'homme. ${ }^{12}$ C'est ce raisonnement qui permet au philosophe de conclure: «Les formes conceptuelles de la pensée qui prédominent dans toutes les époques de la production marchande développée sont issues de rapports de production déterminés » (par l'abstraction-marchandise) ${ }^{13}$. Il s'agit à l'évidence ici de formes de pensée philosophique qui ont émergé dans l'Antiquité grecque ; mais selon l'auteur, cette détermination va plus loin :

8 La division entre travail intellectuel et travail manuel qui règne [dans une société de producteurs de marchandises] a son origine dans le fait que la socialisation est séparée de la production et de la consommation, en d'autres termes dans la séparation de la nature et de la société. Là où des individus pratiquent la production et la consommation comme des activités communautaires [...], le rapport des hommes à la nature forme une unité indissociable avec leurs rapports entre eux. ${ }^{14} \mathrm{Ici}$, même la pensée sociale des hommes est un processus collectif ou coopératif. En revanche, dans les sociétés de production de marchandises, les personnes privées pensent de manière séparée les unes des autres, quoique dans les mêmes formes identiques de la synthèse abstraite. Leur pensée est donc toujours sociale pour la forme, mais il s'agit maintenant d'un pur travail intellectuel, s'appuyant sur une base qui est séparée des activités de la production et de la consommation, c'est-à-dire de toute forme de travail manuel. ${ }^{15}$

Relions ces propositions à l'explication que fournit Nicholas Garnham, en les reformulant légèrement, de façon à mieux saisir les nuances et la complémentarité de ces deux réflexions théoriques. Premièrement, Sohn-Rethel pose l'hypothèse de stades historiques antérieurs (ou à venir), caractérisés par des rapports de production orientés exclusivement vers la satisfaction des besoins des producteurs / consommateurs. En l'absence de l'esclavage, de l'appropriation foncière ou de la systématisation de l'abstraction-marchandise, la forme sociale des rapports matériels de production deviendrait pleinement collective ou coopérative. Il n'existerait pas de séparation de la nature et de la société en ce sens que les rapports matériels de production à partir de la « nature »- c'est-à-dire le processus de transformation de la nature, d'extraction puis de distribution d'un surplus matériel (pour employer la formulation de Garnham) - ne seraient "agrémentés» d'aucun artifice social (esclavage, appropriation foncière, systématisation de l'abstraction-marchandise). Les hommes produiraient de façon « participative » ou « collaborative » (pour utiliser une terminologie plus proche de nous), et même la pensée sociale des hommes deviendrait «un processus collectif ou coopératif »: ils se représenteraient sans «le déni et le mensonge » (pour employer la formulation de Garnham).

A contrario, dans une société de producteurs de marchandises il existe selon Sohn-Rethel, une coupure entre le travail intellectuel (la production culturelle et idéologique dominante) et les autres champs de production (le travail manuel). Cette séparation repose sur le double niveau de médiation que souligne Garnham. D'une part, la forme sociale « économique » occulte et mystifie les relations matérielles de production (à partir 
de ce que la nature fournit); d'autre part les formes culturelles dominantes consacrent une pensée de "personnes privées ", "séparées les unes des autres ", même si ces formes constituent un ensemble social, et demeurent, in fine, déterminées par l'échange marchand et le principe d'identité. L'analyse de Sohn-Rethel les assimile à un "pur travail intellectuel » au même titre que Garnham souligne en quoi la division croissante du travail a pour conséquence l'autonomie relative de la superstructure et le développement de ces formes culturelles spécifiques auxquelles concourent les productions des industries de la culture et de la communication.

\section{Une forme sociale faussement collaborative}

11 Comme le souligne Nicholas Garnham, dans l'extrait reproduit ci-avant, «toute forme de relation sociale étendue dépend de l'extraction et de la distribution d'un surplus matériel et les moyens d'y parvenir constituent donc la caractéristique déterminante de toute formation sociale ». Dans le système des industries de la culture et de la communication, on peut à la limite arguer d'une diversification de ces moyens, avec ce que nous avons appelé par ailleurs un transfert de charges vers les usagers-consommateurs ${ }^{16}$. C'est à ce même phénomène que se réfèrent les analyses critiques $d u$ " travail numérique ", même si les récents travaux de Brice Nixon interrogent avec pertinence le rôle central de ce qu'il nomme « travail du public » (audience labour) pour la valorisation des industries de la culture et de la communication, en proposant qu'il s'agit là d'une tendance lourde et aucunement spécifique à l'émergence des $\operatorname{TICN}^{17}$. Quoi qu'il en soit, les représentations idéologiques du "web collaboratif » ou de l' "économie collaborative » constituent bien des formes culturelles qui restent déterminées par « un système basé sur le contrôle de plus en plus indirect via l'échange de marchandises ${ }^{18}$. Alfred Sohn-Rethel a écrit :

La société gouvernée par la loi économique et l'émergence de la pensée indépendante proviennent toutes deux de la même source. [...] La rationalité de l'intellect indépendant ne peut jamais être plus que la lumière indispensable à l'homme pour vivre dans un monde plongé dans l'obscurité. [...] La division entre la tête et la main et la division de la société en classes ne peuvent pas être supprimés avant que le travail manuel soit socialisé à l'égal de la pensée $\left[. . . .^{19}\right.$

Or, en l'état actuel de développement de nos sociétés, cette socialisation reste encore hypothétique et, quand bien même ce travail d'écriture et mes usages fréquents du dictionnaire de synonymes en ligne du laboratoire $\mathrm{Crisco}^{20}$ constitueraient une preuve de ce décalage entre la tête et la main, rien ne permet de supposer que les pratiques du « web collaboratif » vont dans le sens d'une suppression des divisions susmentionnées. Au contraire, dans la mise en système des industries de la culture et de la communication, nous sommes bien face à une forme sociale faussement participative, comme le souligne par ailleurs Christian Fuchs :

Based on participatory democracy theory, we argue that scholars who suggest that today's Internet is participatory advance an ideology that simply celebrates capitalism without taking into account how capitalist interests dominate and shape the Internet. Web 2.0 is not a participatory system, and it would be better understood in terms of class, exploitation, and surplus value. ${ }^{21}$

14 Les formes culturelles de rapports de production évoquées ci-avant prennent les apparences d'un projet collectif cohérent, basé sur la collaboration libre de producteurs « créatifs » ou « cognitifs » interagissant en réseau, via les TICN. Soulignons, en passant, 
que ces formes culturelles ne sont pas prioritairement destinées à, ni véhiculées par, des travailleurs manuels. Elles sont produites en premier lieu pour et par des travailleurs intellectuels des zones métropolitaines, où elles contribuent objectivement à dissimuler des formes sociales faussement collaboratives de rapports de production. Ceux-ci reposent encore de façon dominante sur l'échange marchand, et permettent encore de façon significative l'extraction de plus-value à des fins de reproduction et d'accumulation du capital. Ces formes culturelles n'ont donc en aucun cas une valeur universelle (même si elles ont potentiellement vocation à s'étendre et à s'appliquer à une diversité de champs socio-économiques). Elles apparaissent cependant à la fois comme un produit et un «ingrédient » important de la phase actuelle du capitalisme, comme un facteur de prolongement du système capitaliste.

\section{L'effectivité matérielle limitée des formes culturelles « collaboratives »}

Retournons brièvement à la question de l'autonomie relative de ces forme culturelles visà-vis de la base des rapports de production matériels, car cette relation est marquée par une certaine ambiguïté. Ces formes culturelles «collaboratives» permettent-elles, superficiellement et sur un mode idéologique, de transfigurer la forme sociale dominante de ces rapports? Songeons à certaines de leurs manifestations les plus abouties, telles que les productions discursives d'un Tim O'Reilly en Amérique du Nord, ou d'un Loïc Le Meur en Europe - que j'ai étudiées à la fois "dans le texte» et au travers d'observations réalisées dans le cadre de salons professionnels organisés par ces deux acteurs : le Web 2.0 Expo de San Francisco (mai 2010) et Le Web 10 à Paris (décembre 2010). Ici, ce que Philippe Bouquillion appelle le "paradigme de la collaboration ${ }^{22}$ semble pleinement se confondre avec sa visée instrumentale, comme l'illustrent ces propositions des « gourous web » Tim O'Reilly et Jon Batelle :

As more and more of our world is sensor-enabled, there will be surprising revelations in how much meaning - and value - can be extracted from their data streams [...]. The new direction for the Web, its collision course with the physical world, opens enormous new possibilities for business, and enormous new possibilities to make a difference on the world's most pressing problems. [...] It's time to leverage the true power of the platform we've built. The Web is no longer an industry unto itself-the Web is now the world. ${ }^{23}$

Cette visée pratique réduit quasiment à néant l'autonomie de la forme culturelle : entièrement tourné vers l'accumulation de plus-value, un tel méta-discours n'a manifestement rien d'une interrogation ou d'une remise en cause des rapports de production, mais il peut en outre difficilement passer pour une transfiguration ou une occultation de ceux-ci (ce en quoi réside une partie de sa faiblesse). Et l'objectif de transformation des hommes et des femmes, à qui cette langue s'adresse et qui la partagent, en " acteurs économiques ", s'avère déjà en grande partie atteint dans ces locus de l'hyper-industrialisation que sont le travail dit numérique, cognitif ou créatif. Les salons professionnels susmentionnés offrent eux-mêmes une sorte de condensé de ce processus général : un public majoritairement composé d' "entrepreneurs » individuels ou de dimension inférieure y échange une part de leur capital (pouvant atteindre plusieurs milliers de dollars, ou d'euros, par personne) non pas tant contre la possibilité de bénéficier des élucubrations idéologiques des gourous, mais avant tout pour pouvoir y effectuer des rencontres qui leur permettront potentiellement d'accroitre leurs gains. De 
grandes firmes des industries de la communication sont présentes, pour des besoins de prospection et de recherche de sous-traitants, mais également en tant que sponsors et fournisseurs de keynote speakers, dans le cadre de transactions win-win avec les firmes organisatrices. Celles-ci perçoivent ainsi une rente reposant in fine sur le travail réel d'hommes et de femmes absents de ce type d'événement, à l'exception du personnel technique, des serveurs et autres hommes-thermos, chargés d'approvisionner les participants en boissons chaudes.

Est-ce à dire que dans cette mise en système étendue des industries de la culture et de la communication, l'infrastructure et la superstructure seraient «fusionnées »? La quasi nullité de l'autonomie de la forme culturelle collaborative signifie-t-elle que le temps et le lieu de la production idéologique coïnciderait pleinement avec le temps et le lieu d'un processus de production matériel, permettant au capital d'accroître sa valeur ${ }^{24}$ ? C'est bien sûr ce que peut laisser penser l'exemple précédent du salon professionnel - à ceci près qu'y rodent les innombrables spectres de producteurs absents. Car comme le rappelle Garnham, l'accumulation du capital nécessite l'intégration de travail vivant au sein du processus de production, en recourant à l'échange argent / force de travail : ce processus « doit combiner ce travail vivant d'une manière déterminée avec du travail objectivé en tant que moyens de production (matériaux bruts et outils), dans la production de marchandises dont l'échange permet de réaliser de la plus-value $»^{25}$

18 Ainsi, l'hypothèse de la fusion semble peu convaincante ${ }^{26}$. La faible autonomie des formes culturelles « collaboratives » apparait au contraire comme le pendant d'une configuration dans laquelle celles-ci s'efforcent de «mimer » leur mutation en formes sociales effectives - vers ce « système opérateur Internet coopératif qui consiste à créer plus de valeur qu'on ne peut capturer », pour reprendre le discours d'o'Reilly. Lorsque nous écrivons que « les dispositifs d'intermédiation sont positionnés "par-dessus" les cycles de production de biens ou de services externes $»^{27}$, c'est pour souligner que les plateformes du « web collaboratif » (une définition étendue inclurait sans nul doute la firme O'Reilly Media), existent en tant que réels parasites sur un processus de production matérielle fondamental, auquel ils doivent en dernière instance leur subsistance. Mais à un second niveau, leur force vient précisément d'une capacité à coordonner et à motiver, via les formes culturelles « collaboratives », des pans de plus en plus importants des travailleurs intellectuels du monde - et donc à garantir effectivement la relative stabilité des relations de production auxquelles participent ces travailleurs.

19 Le « paradigme collaboratif $»^{28}$ est donc à la fois un produit du capitalisme contemporain et un facteur - parmi d'autres - de sa durabilité, de son staying power. Cependant, à l'analyse, il dévoile les fondations artificielles du système des industries de la culture et de la communication. Premièrement, cette mise en système repose bien sur des rapports de production matériels "classiquement» capitalistes, au sein desquels la propriété privée et la division du travail permettent l'extraction de plus-value, à partir de la circulation de ces marchandises clés que sont: les données marketing; les (accès aux) réseaux de communication et espaces de stockage de données ; les équipements informatiques et électroniques ; les programmes, protocoles et contenus protégés par des DPI. Deuxièmement, il ne faut pas perdre de vue le fait que la quasi totalité des activités de production liées aux industries de la culture et de la communication, y compris dans leur mise en système étendue, reposent désormais sur le stockage et la capacité de distribution quasi-instantanée de données numériques, ce qui implique un coût énergétique extrêmement élevé. De récentes études montrent l'ampleur de la dépendance 
de ces industries vis-à-vis des énergies fossiles (notamment les hydrocarbures et le charbon) et fournissent des prévisions alarmantes de la croissance de leurs besoins énergétiques au cours des prochaines décennies ${ }^{29}$. Cette question renvoie bien sûr à l'enjeu plus général de l'impact environnemental des $\mathrm{TICN}^{30}$.

Ces facteurs rendent compte, chacun à leur manière, du caractère profondément artificiel de ces combinaisons de relations et d'activités humaines nommées «industries culturelles et/ou créatives ». Instrument de coordination et de promotion d'activités dont on peut fortement douter de l'utilité sociale, gardien objectif de la perpétuation de l'économie capitaliste et de son exploitation systématique des êtres humains et de l'environnement, cette mise en système apparaît comme une sorte de gigantesque bulle, posée contre un monde réel - qui, malgré tout, continue de rugir et de ramper de l'avant, avec ses antagonismes et l'horizon de leur dépassement.

\section{Retour vers un rêve, poursuivre le projet de la Théorie critique}

21 Saisissons nous, pour conclure, de cette retranscription d'un rêve de Theodor Adorno qui est reproduit en exergue. L'évocation du tramway y fait écho à une curieuse formule glissée dans l'une des dernières lettres envoyées par Adorno à Benjamin, fin février 1940, où il est notamment question de la publication d'extraits des Passagenwerk dans la Zeitschrift für Sozialforschung, ainsi que des recherches entamées avec Horkheimer, qui allaient mener, quelques années plus tard, à la publication de Dialectik der Aufklärung. À cette époque il était encore permis d'espérer que Benjamin rejoindrait prochainement les deux chercheurs aux États-Unis, et de nombreux échanges épistolaires portent sur le problème de l'obtention d'un visa pour ce dernier. Ceci constitue l'une des explications possibles de cette remarque - précédée de commentaires élogieux des sections de l'ouvrage inachevé de Benjamin consacrées à Baudelaire - : « Mais je peux seulement dire avec Max : "Attendons patiemment la réorganisation des tramways". ${ }^{31}$

Qu'on me permette de suggérer, en premier lieu, que l'image de wagons de tramway, accrochés les uns aux autres, puisse symboliser les productions des chercheurs, et que la réorganisation mentionnée renvoie alors à la perspective de collaborations à venir entre ces trois penseurs - et, plus largement, d'une évolution des conditions matérielles et historiques qui grevaient tant leur production durant cette sombre période.

Deuxièmement, considérons l'onirique exergue attribué à un certain Robert August Lange. Cette identité surgie de l'inconscient d'Adorno évoque-t-elle la maison d'édition néerlandaise De Lange, qui a publié de nombreux auteurs interdits en Allemagne nazie, tels que Freud, Brecht ou Kracauer, et qu'il mentionne par ailleurs dans sa correspondance avec Benjamin ? Il s'agit là d'un objet de pure spéculation, et l'important est plutôt le contenu de cette citation rêvée. On sait que la figuration du rêve peut emprunter des représentations connues de la conscience du sujet. Or, comment peut-on analyser cet " amour du sable qui crisse ", entre les roues du wagon et les rails du chemin de fer? C'est dans l'inachevé Passagenwerk que Walter Benjamin a proposé de concevoir la superstructure comme ce qui exprime (ou traduit) la base. Mais Frederic Jameson souligne à juste titre que les termes überbau et basis, employés par Karl Marx, semblent avoir été d'abord utilisés dans le domaine des transports ferroviaires, désignant respectivement le matériel roulant et les voies ${ }^{32}$. La métaphore du "sable qui crisse " 
recouvre dès lors ces singularités infinitésimales, « détails » de processus de médiation, qui contrarient la liaison fonctionnelle et harmonieuse entre überbau et basis; l'autonomie relative des formes culturelles vis-à-vis de la forme sociale "économique » est fonction de la friction qu'exacerbent ces grains de sable qui s'immiscent entre les rails et les roues des wagons.

On peut, en troisième lieu, proposer l'analyse suivante du rêve d'Adorno : ce wagon, qui a l'insolence d'affirmer qu'il roule exclusivement pour l'amour du sable qui crisse, représente la production hypothétique de théoriciens qui affirmeraient inconditionnellement «que la culture donne l'illusion d'une société qui serait digne de l'homme, mais qui n'existe pas; qu'elle dissimule les conditions matérielles sur la base desquelles s'élève toute la vie des hommes; et qu'avec les consolations et les apaisements qu'elle dispense, elle sert à entretenir notre existence dans les mauvaises conditions économiques qui la déterminent $»^{33}$. Pour prolonger la métaphore, cet insolent «amour du sable qui crisse » se rapporterait à une production culturelle au service ultime de l'immobilisation du matériel roulant, et de la dépose des voies, une radicalisation du "thème de la culture comme idéologie, qu'ont d'emblée en commun les théoriciens bourgeois de la violence et leurs contradicteurs, aussi bien Nietzsche que Marx $»^{34}$. Contre ce thème, Adorno formule la proposition suivante :

Identifier la culture au mensonge seulement, voilà qui est excessivement dangereux à un moment où elle tend effectivement à basculer complètement de ce côté et à ne justifier que trop ce genre d'affirmations, de sorte que se trouve compromise toute pensée qui entreprend de résister. Si la réalité matérielle désigne l'univers de la valeur d'échange, et la culture par contre tout ce qui se refuse à accepter la domination de la valeur d'échange, alors il n'est certes pas douteux qu'un tel refus reste illusion aussi longtemps que le monde est comme il est. Mais, comme le principe d'échange libre et équitable est luimême un mensonge, ce qui le nie est du même coup du côté de la vérité : contre le mensonge du monde réifié de la marchandise, on peut encore trouver un correctif dans le mensonge qui en est la dénonciation. Le fait que la culture ait été jusqu'à présent un échec ne justifie pas qu'on contribue encore à son échec, en faisant comme Gribouille qui se jette à l'eau par crainte de se mouiller. Ceux qui sont étroitement liés ensemble ne devraient pas passer sous silence leurs intérêts matériels ni se ravaler au niveau de ces derniers, mais les assumer de façon réfléchie au sein de leur relation pour ainsi les dépasser. ${ }^{35}$

26 Ici, Adorno suggère une alternative dialectique à l'opposition stérile entre la réalité matérielle de la basis et un überbau illusoire, conçue tantôt comme un pur voile idéologique, tantôt comme une réfutation tout aussi intégrale des rapports de production matérielle. La proposition selon laquelle « le principe d'échange libre et équitable est luimême un mensonge " renvoie bien sûr à ce premier niveau d'abstraction que constitue la forme marchandise, dont j'ai souligné plus haut le caractère proprement fondamental en me rapportant aux analyses d'Alfred Sohn-Rethel et de Nicholas Garnham. Mais comme le montre ce dernier, les formes culturelles - qui demeurent intrinsèquement déterminées par des relations de production matérielles - ne peuvent en effet être réduites à de simples représentations idéologiques, ou au mensonge, pour employer le vocabulaire adornien. Les formes culturelles conservent une autonomie relative, mais certaine, vis-àvis de la forme sociale « économique » qui demeure caractéristique de l'infrastructure.

Adorno va dans le même sens lorsqu'il dénonce le caractère erroné d'une conceptualisation du rapport entre forme sociale «économique » et formes culturelles 
qui postulerait que ces dernières puissent s'ériger en bastions souverains d'un refus absolu de la domination de la valeur d'échange. L'insolence des wagons de tramway qui affirment rouler "exclusivement pour l'amour du sable qui crisse » apparaît dès lors comme l'entêtement d'une pensée non-dialectique, qui refuse d'analyser les médiations qui la lient à la base « économique » de la société, qui méconnaît précisément la nécessité de « les assumer de façon réfléchie au sein de leur relation pour ainsi les dépasser ».

Si cette proposition peut à première vue apparaître comme étant elle-même empreinte d'une certaine abstracticité, il convient de se rappeler que cette assomption/analyse des médiations - tentée justement dans l'ouvrage inachevé de Benjamin auquel la citation rêvée renvoie - ne peut se concevoir hors du projet de la théorie critique. Le potentiel que celle-ci entrevoit n'est pas le déraillement spectaculaire des wagons ${ }^{36}$, ni la dépose destructive et jouissive des voies, mais la préfiguration d'une réunion du travail manuel et intellectuel, au sens où le propose Sohn-Rethel ${ }^{37}$. On retrouve cette même proposition essentielle dans un texte de l'ouvrage Prismes, où Adorno affirme :

La culture est le fruit de la séparation radicale entre le travail manuel et le travail intellectuel et tire ses forces de cette séparation qui est en quelque sorte son péché originel. Lorsque la culture se contente de renier cette séparation en donnant l'illusion d'une solidarité directe, elle reste en-dessous de son propre concept. ${ }^{38}$

\section{Conclusion}

Les tensions qui caractérisent les médiations entre les produits de notre activité de chercheurs et les formes sociales des relations de production matérielle peuvent enfin être éclairées par deux observations fortement corrélées. D'une part, il faut prendre la mesure du fait que les moyens de production que nous employons résultent, en dernière instance, d'un processus de socialisation et de redistribution d'un surplus matériel extrait dans le cadre de rapports de production capitalistes, en France et, de façon croissante, dans d'autres régions du monde où l'exploitation du travail offre aux capitalistes des opportunités de valorisation supérieures. D'autre part, il convient de rappeler l'inspiration socialiste d'un des piliers des relations de production spécifiques aux enseignants-chercheurs titulaires français : la conquête, en 1946, du statut de la fonction publique d'État, au sein de laquelle la personne même est qualifiée, par le grade obtenu à l'occasion de concours nationaux. En garantissant l'absence de "marché » du travail, d'employeur et de chômage, ce système préfigure explicitement un mode de relations de production débarrassé de l'exploitation capitaliste ${ }^{39}$.

31 Cet apparent détail paraît d'autant plus significatif lorsqu'on aborde l'analyse de médiations entre l'infrastructure et ces formes culturelles auxquelles nous sommes amenés à contribuer, qui requièrent de plus en plus d'éléments discursifs censés attester de nos capacités de managers de formations, ou de nos compétences de techniciens et producteurs d'information stratégique ${ }^{40}$. "La valeur de l'État en tant qu'arme du prolétariat dépend de ce que le prolétariat saura en faire » écrivait Georg Lukács ${ }^{41}$, en des temps qui ne sont pas si éloignés. Une analyse ignorant l'évolution des perspectives théoriques matérialistes concernant l'État (et leur effectivité dans certains contextes historiques) serait bien en peine de concevoir le rôle qu'elles peuvent encore jouer, en tant que facteurs de renforcement d'une autonomie effective de ces formes culturelles singulières que sont les produits des sciences sociales. 


\section{BIBLIOGRAPHIE}

Adorno T.W. (1986), Prismes: Critique de la culture et société, Paris, Payot.

Adorno T.W. (2003), Minima Moralia. Réflexions sur la vie mutilée. Paris, Payot

Adorno T.W. (2007), Mes rêves, Paris, Stock.

Adorno T. W., Benjamin W. (2006), Correspondance Adorno-Benjamin: 1928-1940, H. Lonitz (ed.), Paris, Gallimard.

Andrejevic M. (2009), « Exploiting Youtube: Contradictions of user-generated labor » In The Youtube Reader, 406-424.

Batelle J., O'reilly T. (2009), Web Squared: Web 2.0 Five Years On (vol. 20, No. 1), Sebastopol, O'reilly Media.

Bouquillion P., Matthews J. T. (2010), Le web collaboratif: mutations des industries de la culture et de la communication, Grenoble, P.U.G.

Bouquillion P., Matthews J. T. (2012), « Collaborative Web And The Cultural Industries System: A Critical Appraisal », Disponible en ligne : http://www.observatoire-omic.org/fr/art/497/ collaborative-web-and-the-cultural-industries-system-a-critical-appraisal.html

Bouquillion P. (2013), « Socio-économie des industries culturelles et pensée critique : le web collaboratif au prisme des théories des industries culturelles » in Les enjeux de l'information et de la communication. Supplément 2013a, «Contributions aux recherches critiques sur la communication ».

Bouquillion P., Miège B., Moeglin P. (2013), L'industrialisation des biens symboliques: les industries créatives en regard des industries culturelles, Grenoble, P.U.G.

Casilli A., Carton D. (2015), Qu'est-ce que le digital labor?, Editions de l'Ina.

Comor E. (2010), « Digital prosumption and alienation » In Ephemera, 10(3/4).

Flipo F., Dobré M., Michot M. (2013), La face cachée du numérique. L'impact environnemental des nouvelles technologies. Paris, Éditions L'échappée.

Friot B. Vaincre Macron. Paris, La Dispute, 2017.

Fuchs C. (2013), «Class and exploitation on the internet » in T. Scholz, (dir.) (2013). Digital labor: the internet as playground and factory. Londres, Routledge, 211-224.

Garnham N. (1979), « Contribution to a political economy of mass-communication » In Media, Culture \& Society. 1(2), 123-146.

Gibson, C., Klocker, N. (2004), « Academic publishing as 'creative' industry, and recent discourses of 'creative economies' : some critical reflections ", in Area, 36(4), 423-434.

Hesmondhalgh D. (2010), « User-generated content, free labour and the cultural industries » in Ephemera, 10(3/4), 267-284.

Jameson F. (1991), Postmodernism, or, the cultural logic of late capitalism. Durham, Duke University Press. 
Lovink, G., Ross, A. (2007), « Organic intellectual work : interview with Andrew Ross », In Lovink, G. \& Rossiter, N. (dir.). Mycreativity Reader: a critique of creative industries, 227-237.

Lukács G. (1965 [1924]). Lénine, Paris, Études Et Documentation Internationales.

Matthews J. T., Rouzé V., Vachet J. (2014), La culture par les foules ? Le crowdfunding et le crowdsourcing en questions, Paris, MKF Éditions.

Matthews J. T., Vachet J. (2014a), « Le crowdsourcing et le crowdfunding culturels dans le web collaboratif ». Suite Numérique $\mathrm{N}^{\circ} 3$, in Matthews J. T., Rouzé V., Vachet J., La culture par les foules ? Le crowdfunding et le crowdsourcing en questions, Paris, MKF Éditions, 28-39.

Matthews J. T., Vachet J. (2014b), « La production et le financement collaboratifs : vers une extension de l'industrialisation culturelle? ». Suite Numérique $\mathrm{N}^{\circ} 4$, in Matthews J. T., Rouzé V., Vachet J., La culture par les foules ? Le crowdfunding et le crowdsourcing en questions, Paris, MKF Éditions, 40-55.

Matthews J.T. (2014), Un parcours de recherche au croisement de la théorie critique et des approches socio-économiques des industries culturelles. Bilan et perspectives. Habilitation à diriger les recherches en sciences de l'information et de la communication, Université Toulouse Jean Jaurès.

Means, A. (2011). «Creativity as an educational problematic within the biopolitical economy ", in Peters, M. A., \& Bulut, E. (dir.). Cognitive capitalism, education, and digital labor. Peter Lang, 211-228.

Mills M.P. (2013), The Cloud Begins With Coal : Bid Data, Big Networks, Big Infrastructure And Big Power, An Overview Of The Electricity Used By The Global Digital Ecosystem, National Mining Association / American Coalition For Clean Coal Electricity.

Nixon B. (2014), « Toward a political economy of 'audience labour' in the digital era ", in Triple C, 12(2), 713-734.

Terranova T. (2000), «Free labor: producing culture for the digital economy », in Social Text, 18 (2), 33-58.

Peters M. A., Bulut E. (dir.) (2011), Cognitive capitalism, education, and digital labor. New York, Peter Lang.

Sohn-Rethel A. (2010), La Pensée-marchandise. Paris, Éditions du Croquant.

Scholz T. (dir.) (2012), Digital labor: the internet as playground and factory. Londres, Routledge.

Taylor P. (2013), « Putting theory to work - a.k.a 'if you don't like academia, why don't you leave?' ", in Ephemera, 13(4), 267-284.

Williams R. (1973), « Base and superstructure in marxist cultural theory », in New Left Review, 82 (10), 130-143.

\section{NOTES}

1. Voir P. Bouquillion et J. Matthews, Le web collaboratif: mutations des industries de la culture et de la communication, P.U.G., 2010 et P. Bouquillion et J. Matthews, "Collaborative web and the cultural industries system : a critical appraisal », 2012, disponible en ligne : http://www.observatoireomic.org/fr/art/497/collaborative-web-and-the-cultural-industries-system-a-criticalappraisal.html

2. J. Matthews, V. Rouzé, J. Vachet, La culture par les foules? Le crowdfunding et le crowdsourcing en questions, MKF éditions, 2014. 
3. C'est à dessein que je n'emploie pas ici la notion de «contenu»: les productions de ces industries ne sont pas contenues dans les «contenus"; le travail des usagers, des publics, consiste précisément à parachever ces productions, au travers de ce qu'on a longtemps désigné sans doute à tort - par le terme « consommation ».

4. R. Williams, « Base and superstructure in Marxist cultural theory » in New Left Review, n82, vol 10, 1973, pp. 130-143.

5. N. Garnham, «Contribution to a political economy of mass-communication » in Media, Culture \& Society, volume $1, n^{\circ} 2,1979$, pp. 123-146.

6. La liste ci-après n'est évidemment pas exhaustive, mais on peut mentionner, entre autres, les contributions suivantes à ce débat: M. Andrejevic, « Exploiting YouTube: Contradictions of usergenerated labor » in The YouTube Reader, 2009 ; T. Terranova, « Free labor: Producing culture for the digital economy " in Social text, volume 18, $n^{\circ} 2,2000$; E. Comor, «Digital prosumption and alienation » in Ephemera, volume 10, n³-4, 2010 ; D. Hesmondhalgh «User-generated content, free labour and the cultural industries » in Ephemera, volume 10, n³-4, 2010 ; M. Peters et E. Bulut (dir.), Cognitive capitalism, education, and digital labor. Peter Lang, 2011 ; T. Scholz (dir.), Digital labor: The Internet as playground and factory, Routledge, 2013 ; A. Casilli et D. Carton, Qu'est-ce que le digital labor?, Editions de l'INA, 2015.

7. N. Garnham, op. cit., p. 129. Traduction en français : «Dans la mesure où le matérialisme historique est matérialiste, il est basé sur les postulats que souligne Williams. Mais dans la mesure où il est historique, il est préoccupé par l'analyse des modes fluctuants de cette relation matérielle fondamentale, qui sont tous des formes de cette relation. En particulier, il est postulé que toute forme de relation sociale étendue dépend de l'extraction et de la distribution d'un surplus matériel et les moyens d'y parvenir constituent donc la caractéristique déterminante de toute formation sociale. Ainsi, on doit distinguer entre deux types de forme, une forme sociale qui est une série de relations qui, dans la mesure où elles opèrent inconsciemment, peuvent être abstraitement analysées et déterminées avec la précision d'une science naturelle, et une forme culturelle qui, tout en impliquant un support matériel, n'est pas elle-même matérielle et qui a une relation essentiellement médiate avec la réalité matérielle qu'elle représente. En effet, il existe une séparation essentielle entre ces domaines formels distincts, séparation qui permet à l'idéologie d'entrer, parce qu'elle permet le déni et le mensonge, qui dépendent tous deux sur une relation qui n'est pas déterminante. Toutefois, cette autonomie est acquise au prix d'une perte d'effectivité réelle ou matérielle. Les formes culturelles ne deviennent effectives que lorsqu'elles sont traduites en formes sociales qui ont une effectivité matérielle. Pour retourner au niveau des formes sociales, l'économique est une forme historiquement spécifique des relations sociales de production et de distribution. C'est la forme que ces relations prennent au sein d'une formation sociale dans laquelle domine l'échange de marchandises. Ce que souligne Marx dans Le Capital, c'est que la réelle transition historique vers le capitalisme implique une évolution d'un système de relations sociales et de domination basé sur le contrôle physique direct de propriété foncière et de personnes, vers un système basé sur le contrôle de plus en plus indirect via l'échange de marchandises, et en particulier, via l'échange de la marchandise que constitue la force de travail. Ce processus historique réel est un processus réel d'abstraction sociale dont l'analyse requiert une abstraction théorique appropriée. C'est l'existence réelle de ce niveau économique abstrait de production marchande étendue qui permet le développement d'une division du travail croissante et, par conséquent, pour le développement des formes superstructurelles spécifiques du capitalisme. Ainsi, l'autonomie relative de la superstructure est une caractéristique réelle et de plus en plus centrale du capitalisme, mais elle est elle-même déterminée au niveau de l'économique, et constitue en dernière instance une forme, médiate à deux niveaux, d'une relation matérielle qui reste également déterminante dans et via l'économique. » 
8. Garnham précise toutefois (p. 127) : «The pertinence or meaning of any analytical category, such as base and superstructure, expressing as it does a relationship, will shift as the historical reality it is used to explain shifts. Similarly, we could say that the purpose of a political economy of culture is to elucidate what Marx and Engels meant in the German Ideology by 'control of the means of mental production', while stressing that the meaning that they gave to the term was quite clearly historical and therefore shifting and was never meant to be frozen into some simple dichotomy as it has so often been in subsequent Marxist writing.» («La pertinence ou la signification de toute catégorie analytique, telle que base et superstructure, en exprimant comme elle le fait une relation, évoluera à mesure qu'évolue la réalité historique qu'elle vise d'expliquer. De façon semblable, nous pourrions dire que le but d'une économie politique de la culture est d'élucider ce que Marx et Engels entendaient dans l'Idéologie allemande par "le contrôle des moyens de production mentale", tout en signalant que la signification qu'ils ont donnée à ce terme était très clairement historique et par conséquent évolutive, et n'avait pas vocation à être figée dans une dichotomie simple comme cela a été si souvent le cas dans l'écriture marxiste ultérieure. »)

9. Ibid, p. 133.

10. A. Sohn-Rethel, La pensée-marchandise, Éditions du Croquant, 2010, pp. 39-78.

11. Il ne se réfère pas ici au procès de production de biens, qui ne se caractérise pas dans les stades historiques antérieures au capitalisme par l'échange de la marchandise force de travail contre la marchandise monnaie (salaire), celui-ci devenant par contre la forme sociale dominante à partir du 19ème siècle.

12. Comme on le sait, d'après la notion de caractère fétiche de la marchandise, cette «abstracticité » de la marchandise occulte à ses possesseurs le processus matériel et historique de la formation des marchandises, empêchant à l'homme de voir qu'une société où la circulation des marchandises constitue le lien entre toutes les choses (matérielles) est une société abstraite. Mais bien que Sohn-Rethel n'entend aucunement nier cette proposition centrale ce n'est pas sur ce niveau d'analyse que porte sa démonstration.

13. Op. cit., p. 66.

14. Il entend par «lien social fondé sur la production» une «coopération (ayant) lieu entre individus agissant de leur propre gré et sur la base de la propriété collective de leurs moyens de production. [...] Quand ce lien inclut la totalité ou la quasi-totalité de la production sociale [...] nous pouvons parler d'unité sociale de la tête et de la main, donc de communisme.» (op. cit., p. 147)

15. Ibid.

16. J. Matthews, J. Vachet., «Le crowdsourcing et le crowdfunding culturels dans le web collaboratif ». Suite numérique $\mathrm{n}^{\circ} 3$, in J. Matthews, V. Rouzé, J. Vachet, La culture par les foules ? Le crowdfunding et le crowdsourcing en questions, Paris, MKF Éditions, p. 32.

17. B. Nixon, «Toward a Political Economy of 'Audience Labour' in the Digital Era », in Triple C, volume 12, n 2, 2014, pp. 713-734.

18. N. Garnham, op. cit., p. 129.

19. Sohn-Rethel, op. cit., pp 144-145.

20. http://www.crisco.unicaen.fr/des/ consulté le 30/01/2018.

21. C. Fuchs, «Class and exploitation on the Internet » in T. Scholz (dir.), Digital labor: The Internet as playground and factory, Routledge, 2013, p. 215. Traduction: «À partir de la théorie de la démocratie participative, nous affirmons que les chercheurs qui suggèrent que l'Internet contemporain est participatif véhiculent une idéologie qui ne fait que célébrer le capitalisme sans prendre en considération la manière dont les intérêts capitalistes dominent et formatent l'Internet. Le web 2.0 n'est pas un système participatif, et il serait mieux compris en termes de classe, d'exploitation et de plus-value. » 
22. P. Bouquillion, "Socio-économie des industries culturelles et pensée critique : le Web collaboratif au prisme des théories des industries culturelles » in Les Enjeux de l'information et de la communication. Supplément 2013 A.

23. J. Batelle, T. O'Reilly,Web squared: Web 2.0 five years on, Sebastopol, O'Reilly Media, 2009, p. 12. Traduction : "Alors que de plus en plus de notre monde est équipé de capteurs de données, il y aura des révélations surprenantes sur la quantité de significations - et de valeur - pouvant être extraite des flux de données (...). La nouvelle direction pour le web, sa collision avec le monde physique, ouvre d'énormes nouvelles possibilités pour le commerce, et d'énormes nouvelles possibilités d'apporter des solutions aux problèmes les plus pressants du monde. Il est temps d'exploiter le véritable pouvoir de la plateforme que nous avons construite. Le web n'est plus une industrie circonscrite - le web est maintenant le monde. »

24. Garnham, op. cit., p. 139.

25. Ibid., p. 140.

26. Voir P. Bouquillion, J. Matthews, op. cit., 2012 et J. Matthews, J. Vachet, « La production et le financement collaboratifs: vers une extension de l'industrialisation culturelle?». Suite numérique $\mathrm{n}^{\circ} 4$, in J. Matthews, V. Rouzé, J. Vachet, La culture par les foules? Le crowdfunding et le crowdsourcing en questions, MKF Éditions, 2014, pp. 40-55.

27. J. Matthews, J. Vachet., «Le crowdsourcing et le crowdfunding culturels dans le web collaboratif ». Suite numérique $\mathrm{n}^{\circ} 3$, in J. Matthews, V. Rouzé, J. Vachet, La culture par les foules ? Le crowdfunding et le crowdsourcing en questions, Paris, MKF Éditions, p. 36.

28. P. Bouquillion, B. Miège, P. Mœglin, L'industrialisation des biens symboliques: les industries créatives en regard des industries culturelles, PUG, 2013.

29. M.P. Mills, The Cloud Begins with Coal : Bid Data, Big Networks, Big Infrastructure and Big Power, an Overview of the Electricity Used By the Global Digital Ecosystem, National Mining Association / American Coalition for Clean Coal Electricity, 2013.

30. F. Flipo, M. Dobré, M. Michot, La face cachée du numérique. L'impact environnemental des nouvelles technologies, Éditions L'Échappée, 2013.

31. T. W. Adorno, W. Benjamin, Correspondance Adorno-Benjamin: 1928-1940, H. Lonitz (Ed.), Gallimard., 2006, p. 369.

32. F. Jameson, Postmodernism, Or, the Cultural Logic of Late Capitalisme. Durham, Duke University Press, 1991, p. 46.

33. T. W. Adorno, Minima Moralia. Réflexions sur la vie mutilée, Payot 2003, p. 54.

34. Ibid.

35. Ibid., pp. 55-56.

36. Méditons la remarque qu'Adorno adresse à Benjamin dans cet extrait d'une lettre envoyée en mai 1938, où l'on trouve une autre métaphore se rapportant au domaine des transports, et pour laquelle je suggère de garder à l'esprit le fait que « l'équipage » a pour fonction de maintenir le navire sur les rails imaginaires qui le dirigent vers sa destination : « Avec un art consommé, on détache un bateau de sauvetage; mais l'art consiste principalement à le remplir d'eau pour l'amener à chavirer. Pour ma part, j'estime avec vous que les chances sont meilleures, quand tout le bateau fait naufrage corps et bien, de voir subsister quelque chose sinon de l'équipage du moins du fret. » (T. W. Adorno, W. Benjamin, op. cit., p. 286.)

37. A. Sohn-Rethel, op. cit, p. 147.

38. T. W. Adorno, Prismes : critique de la culture et société, Payot, 1986, p. 15.

39. B. Friot, Vaincre Macron, La Dispute, 2017.

40. Voir, sur ce point, les apports des travaux suivants: C. Gibson, N. Klocker, "Academic publishing as 'creative' industry, and recent discourses of 'creative economies' : some critical reflections » in Area, volume 36, $\mathrm{n}^{\circ}$ 4, 2004, pp. 423-434. ; G. Lovink, A. Ross, « Organic intellectual work: interview with Andrew Ross ", in G. Lovink, N. Rossiter (dir.), MyCreativity Reader: A Critique of Creative Industries, 2007, pp. 227-237 ; J. Matthews, Un parcours de recherche au 
croisement de la théorie critique et des approches socio-économiques des industries culturelles. Bilan et perspectives. Habilitation à diriger les recherches en Sciences de l'information et de la communication, Université Toulouse - Jean Jaurès, 2014 ; A. Means, «Creativity as an Educational Problematic within the Biopolitical Economy » in M. A. Peters, E. Bulut (dir.), Cognitive capitalism, education, and digital labor. Peter Lang, 2011, pp. 211-228 ; P. Taylor, « Putting theory to work a.k.a 'if you don't like academia, why don't you leave?' » in Ephemera, volume 13, n 4, 2013, pp. 267-284.

41. G. Lukács, Lénine, Études et documentation internationales, 1965 [1924].

INDEX

Mots-clés : Industries culturelles, web collaboratif, digital labour, Nicholas Garnham, Theodor W. Adorno

\section{AUTEUR}

\section{JACOB MATTHEWS}

Professeur des Universités, Paris 8; directeur du celti - Paris 8 